\title{
Thermodynamic and magnetic properties of the layered triangular magnet $\mathrm{NaNiO}_{2}$
}

\author{
P. J. Baker, ${ }^{1}$ T. Lancaster, ${ }^{1}$ S. J. Blundell, ${ }^{1}$ M. L. Brooks, ${ }^{1}$ W. Hayes,${ }^{1}$ D. Prabhakaran, ${ }^{1}$ and F. L. Pratt ${ }^{2}$ \\ ${ }^{1}$ Clarendon Laboratory, University of Oxford, Parks Road, Oxford OX1 3PU, United Kingdom \\ ${ }^{2}$ ISIS Muon Facility, ISIS, Chilton, Oxon. OX11 OQX, United Kingdom
}

(Received 30 May 2005; revised manuscript received 19 July 2005; published 8 September 2005)

\begin{abstract}
We report muon-spin rotation, heat capacity, magnetization, and ac magnetic susceptibility measurements of the magnetic properties of the layered spin-1/2 antiferromagnet $\mathrm{NaNiO}_{2}$. These show the onset of long-range magnetic order below $T_{\mathrm{N}}=19.5 \mathrm{~K}$. Rapid muon depolarization, persisting from $T_{\mathrm{N}}$ to about $5 \mathrm{~K}$ above $T_{\mathrm{N}}$, is consistent with the presence of short-range magnetic order. The temperature and frequency dependence of the ac susceptibility suggests that magnetic clusters persist above $25 \mathrm{~K}$ and that their volume fraction decreases with increasing temperature. A frequency dependent peak in the ac magnetic susceptibility at $T_{\text {sf }}=3 \mathrm{~K}$ is observed, consistent with a slowing of spin fluctuations at this temperature. A partial magnetic phase diagram is deduced.
\end{abstract}

DOI: 10.1103/PhysRevB.72.104414

PACS number(s): 76.75.+i, 75.50.Ee, 75.30.Gw

\section{INTRODUCTION}

Triangular lattice antiferromagnets exhibit a rich variety of ordering phenomena related to geometrical frustration. ${ }^{1}$ When the triangles forming the lattice are distorted from equilateral to isosceles there is a partial release of the geometrical frustration, which can lead to more unusual forms of magnetic order exemplified in the fractional spin liquid in $\mathrm{Cs}_{2} \mathrm{CuCl}_{4},{ }^{2}$ the spin-density wave in $\mathrm{Na}_{0.75} \mathrm{CoO}_{2},{ }^{3}$ and the rich $B$ - $T$ phase diagram of $\mathrm{CoNb}_{2} \mathrm{O}_{6} \cdot{ }^{4}$ The series of triangular lattice magnets $\mathrm{LiNiO}_{2}, \mathrm{AgNiO}_{2}$, and $\mathrm{NaNiO}_{2}$, seems to offer the possibility of tuning the release of the geometrical frustration by changing the exchange parameters, allowing models describing these systems ${ }^{5-7}$ to be tested experimentally. However, the difficulty of producing stoichiometric $\mathrm{LiNiO}_{2}$ has led to a variety of sample-dependent results. ${ }^{8}$ $\mathrm{AgNiO}_{2}$ can be produced in stoichiometric form but no magnetic Bragg peaks have so far been reported. ${ }^{9}$ Recently neutron powder diffraction studies have determined the low temperature magnetic structure of $\mathrm{NaNiO}_{2} \cdot{ }^{10,11}$ A peak in the magnetic susceptibility interpreted as the Néel temperature, $T_{\mathrm{N}}$, has been observed around $20 \mathrm{~K}^{.12-14}$ These disagree with the value of $T_{\mathrm{N}} \sim 23 \mathrm{~K}$ recently deduced from neutron powder diffraction data ${ }^{11}$ (see below). In this paper, we report a detailed study of polycrystalline $\mathrm{NaNiO}_{2}$ establishing the value of $T_{\mathrm{N}}=19.5 \mathrm{~K}$ from both bulk and microscopic techniques (zero-field muon spin rotation $(\mu \mathrm{SR})$, heat capacity, and magnetic susceptibility measurements). Our work demonstrates the presence of magnetic clusters forming above $T_{\mathrm{N}}$. We also investigate the low-temperature anomaly in the magnetic susceptibility observed previously. ${ }^{11}$

\section{CRYSTAL AND MAGNETIC STRUCTURE}

Above $480 \mathrm{~K}$ the space group of $\mathrm{NaNiO}_{2}$ is rhombohedral $(R \overline{3} m)$ and there is a cooperative Jahn-Teller transition to a low temperature monoclinic $(\mathrm{C} 2 / \mathrm{m})$ phase below this temperature. ${ }^{15}$ The low-temperature structure can be considered to be layers of $\mathrm{NiO}_{6}$ octahedra in the $a b$ plane, with a trigonal distortion lengthening the $\mathrm{Ni}-\mathrm{O}$ bonds in the $a c$ plane along an axis at $41^{\circ}$ to the $c$ axis of the crystal. The $\mathrm{Ni}^{3+}\left(3 d^{7}\right)$ ion is in the low spin state $\left(t_{2 g}^{6} e_{g}^{1}, S=1 / 2\right)$, so the ground state is a singly occupied $\left|3 z^{2}-r^{2}\right\rangle$ orbital with $z$ along the axis of the Jahn-Teller induced trigonal distortion. ${ }^{15}$

Darie et al. ${ }^{10}$ find the ordering of the magnetic moments at $4 \mathrm{~K}$ to be a slight modification of the A-type antiferromagnetic ordering previously proposed. ${ }^{12}$ The magnetic moments were found to be aligned at an angle of $100(2)^{\circ}$ to the $a$ axis in the $a c$ plane with no moment along the $b$ axis. The CurieWeiss constant, $\theta_{\mathrm{CW}}=+36 \mathrm{~K},{ }^{14}$ shows the presence of ferromagnetic interactions above $T_{\mathrm{N}}$.

The intralayer and interlayer exchange constants of $\mathrm{NaNiO}_{2}, J_{\|}=-13.3 \mathrm{~K}$ and $J_{\perp}=1.3 \mathrm{~K}$, have been determined from a model assuming an A-type antiferromagnetic ordering in the presence of anisotropy; ${ }^{16}$ the layers are sufficiently strongly coupled to permit long range magnetic order below $T_{\mathrm{N}}$. The Ni-O-Ni bond angles are $\approx 95^{\circ}$ at room temperature. ${ }^{15}$ An undistorted $90^{\circ}$ geometry favours weak ferromagnetic superexchange, while a large deviation from a $90^{\circ}$ bond angle can reverse the sign of this exchange coupling. ${ }^{17}$ In $\mathrm{NaNiO}_{2}$ it appears that despite the distortion, in-plane ferromagnetic coupling prevails, though the precise nature of the spin and orbital ordering remains under discussion..$^{5-7}$

\section{EXPERIMENTAL RESULTS}

$\mathrm{NaNiO}_{2}$ was prepared from $\mathrm{Na}_{2} \mathrm{O}_{2}$ and $\mathrm{NiO}$ powders heated at $700{ }^{\circ} \mathrm{C}$ for $100 \mathrm{~h}$ under pure oxygen flow, with intermediate grinding. X-ray powder diffraction showed that the impurity concentration was below the $2 \%$ resolution limit of the apparatus. Heat capacity data measured in magnetic fields between 0 and $14 \mathrm{~T}$, taken with a Quantum Design PPMS, are shown in Fig. 1(a). In zero field the transition at $T_{\mathrm{N}}=19.5 \mathrm{~K}$ is seen as a rather broad peak, and there is no evidence for other phase transitions below $30 \mathrm{~K}$. With increasing magnetic field the temperature of this peak decreases to about $14 \mathrm{~K}$ [see Fig. 1(a)]. 


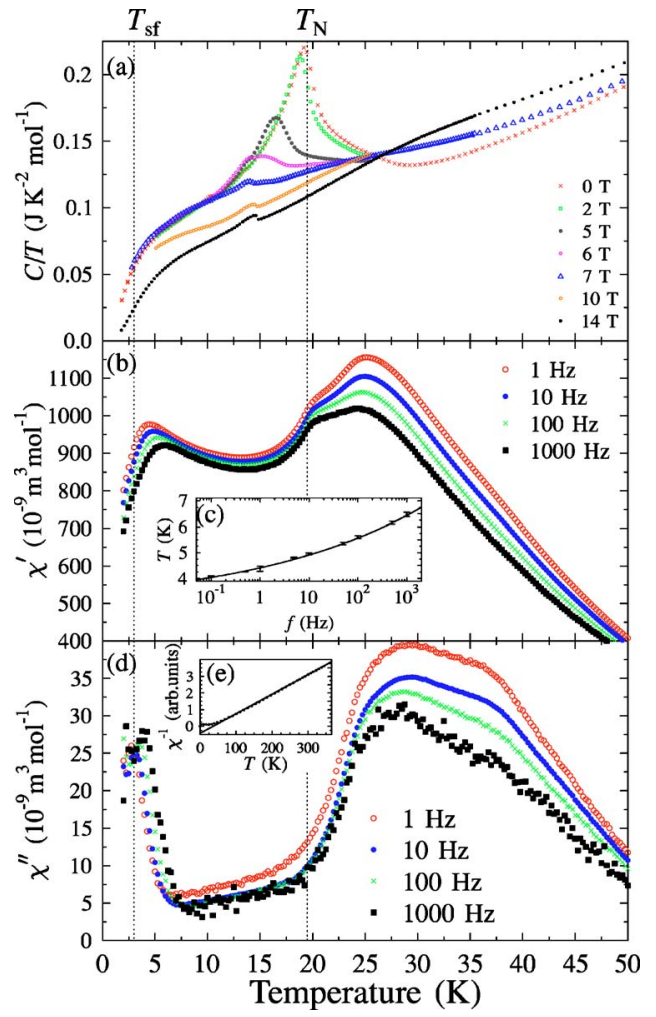

FIG. 1. (Color online) The panels correspond to: (a) Heat capacity divided by temperature in fields between 0 and $14 \mathrm{~T}$. (b) Real part, $\chi^{\prime}$, of the ac magnetic susceptibility. (c) Temperature dependence of the peak in $\chi^{\prime}$ associated with $T_{\text {sf }}$ with a fit to the Ogielski relation [Eq. (1)]. (d) Imaginary part, $\chi^{\prime \prime}$, of the ac magnetic susceptibility. (e) Inverse of magnetic susceptibility data against temperature with a linear fit showing the high-temperature Curie-Weiss behavior. The vertical dashed lines indicate temperatures referred to in the text.

Magnetic susceptibility data, taken using a Quantum Design MPMS superconducting quantum interfence device (SQUID) magnetometer are shown in Figs. 1(b), 1(d), and 1(e). The high temperature dc susceptibility data, shown in Fig. 1(e), are consistent with $\theta_{\mathrm{CW}}=+36 \mathrm{~K}^{14}$ The real and imaginary parts of the ac susceptibility, $\chi^{\prime}$ and $\chi^{\prime \prime}$, are presented in Figs. 1(b) and 1(d) (driving field 3.5 Oe). They show a frequency dependent peak in $\chi^{\prime}$ located slightly above a spin-freezing temperature $T_{\text {sf }}$, which is determined below. Figure 1(d) shows the temperature variation of this peak in $\chi^{\prime}$ with frequency. It was found that this could be fitted to the Ogielski scaling relation ${ }^{18}$

$$
T=T_{\text {sfL }}\left[1+\left(f \tau_{0}\right)^{1 / z \nu_{c}}\right]
$$

where $T$ is the temperature of the peak in $\chi^{\prime}, f$ is the measurement frequency, $\tau_{0}$ is the relaxation time of the system, $z$ is a dynamic exponent, and $\nu_{c}$ is a critical exponent. Fitting the data to Eq. (1) gives $T_{\text {sf }}=3 \pm 0.2 \mathrm{~K}, \tau_{0}=5.4(2) \times 10^{-3} \mathrm{~s}$ and $z \nu_{c}=8.1 \pm 0.4$. This is typical of glassy behavior or the slowing of spin fluctuations. At $T_{\mathrm{N}}, \chi^{\prime}$ has a small maximum and rises to a larger peak near $25 \mathrm{~K}$, with the frequency dependence increasing from $T_{\mathrm{N}}$ to the peak, and decreasing above it. The temperature of the peak in $\chi^{\prime}$ at $25 \mathrm{~K}$ decreases

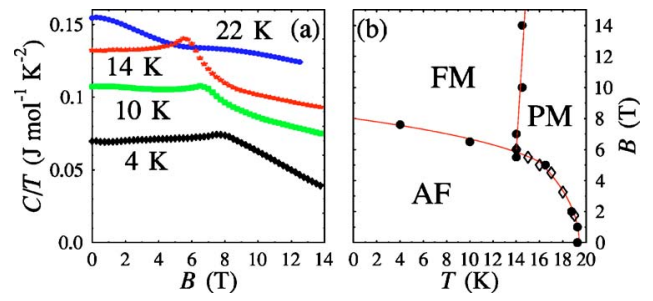

FIG. 2. (Color online) (a) Heat capacity divided by temperature vs field at four temperatures. (b) Partial magnetic phase diagram deduced from heat capacity $(\bullet)$ and magnetization $(\diamond)$ data. AF: A-type antiferromagnetic phase. PM: Paramagnetic phase. FM: Ferromagnetic phase.

slowly with increasing frequency, which may be related to the presence of two sets of relaxation times varying differently with temperature (see below). $\chi^{\prime \prime}$ also rises sharply near $25 \mathrm{~K}$ but to a plateau continuing up to $\sim 35 \mathrm{~K}$. Together these suggest that short-range order persists up to $\sim 25 \mathrm{~K}$, that slowly fluctuating clusters of spins are present within a fast fluctuating paramagnetic bulk above this temperature, and that the volume fraction of clusters decreases with increasing temperature.

Constant temperature heat capacity data with varying magnetic field are presented in Fig. 2(a). Except for the data taken at $22 \mathrm{~K}$, a peak is observed which corresponds to the field labeled $H_{\mathrm{C} 1}$ in the magnetization data reported in Ref. 16. This suggests that this marks the upper field boundary of A-type antiferromagnetic order. At $22 \mathrm{~K}$ the heat capacity decreases with increasing field consistent with short-range order. The partial magnetic phase diagram deduced from our heat capacity and magnetization data is shown in Fig. 2(b).

Our zero-field $\mu \mathrm{SR}$ experiments were carried out using the DOLLY instrument at the Paul Scherrer Institute (PSI), Villigen, Switzerland. In our $\mu$ SR experiments, spin polarized positive muons $\left(\mu^{+}\right.$, mean lifetime $2.2 \mu \mathrm{s}$, momentum $28 \mathrm{MeV} / c$ ) were implanted into polycrystalline $\mathrm{NaNiO}_{2}$. The decay positron asymmetry function, $A(t),{ }^{19}$ is proportional to the average spin polarization of the muons stopped within the sample. The muon spin precesses around an internal magnetic field, $B_{\mu}$, at a frequency $\nu_{\mu}=\left(\gamma_{\mu} / 2 \pi\right)\left|B_{\mu}\right|$, where $\gamma_{\mu} / 2 \pi=135.5 \mathrm{MHz} \mathrm{T}^{-1}$.

The asymmetry data were fitted to Eq. (2) (Ref. 20) below $T_{\mathrm{N}}$, and to Eq. (3) above $T_{\mathrm{N}}$ :

$$
\begin{gathered}
A(t)=A(0)\left[P_{1} e^{-\lambda_{1} t}+P_{2} e^{-\lambda_{2} t} \cos \left(2 \pi \nu_{\mu} t+\phi_{0}\right)\right], \\
A(t)=A(0)\left(P_{\mathrm{f}} e^{-\lambda_{\mathrm{f}} t}+P_{\mathrm{s}} e^{-\lambda_{\mathrm{s}} t}\right),
\end{gathered}
$$

where $A(0)$ is the initial asymmetry. $P_{1}$ and $P_{2}$ are, respectively, the longitudinal and transverse components of the muon polarization, and $P_{1}+P_{2}=1$. The exponential relaxation associated with $P_{1}$ reflects the dynamical fluctuations of the fields being probed. The $P_{2}$ term describes muon precession with a distribution of local fields dephasing the muon spins. In a fully magnetically ordered polycrystalline sample we expect $P_{2} / P_{1}=2$. Coherent muon precession will be observed if long range order is present within the sample. $P_{\mathrm{s}}$ and $P_{\mathrm{f}}$ describe slow and fast dynamic fluctuations, respec- 


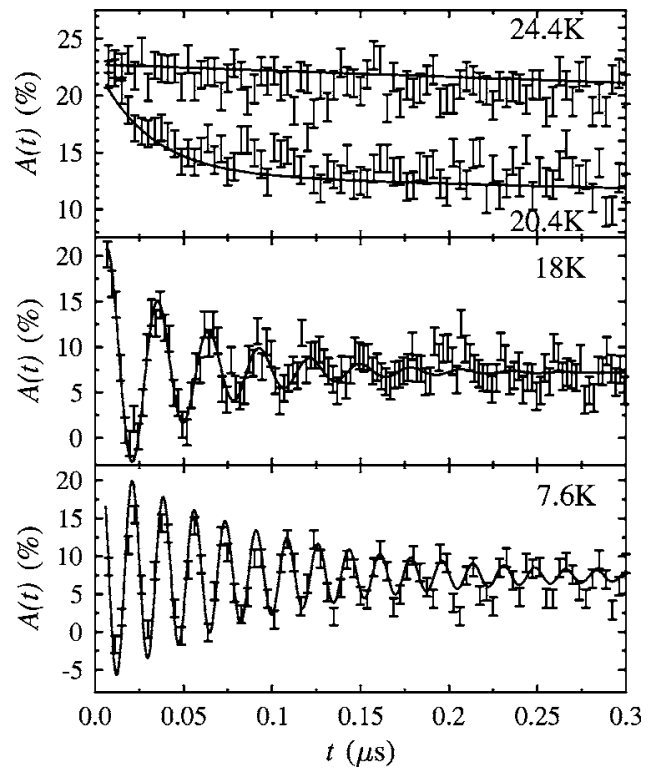

FIG. 3. Muon decay asymmetry in $\mathrm{NaNiO}_{2}$ plotted at different temperatures. The solid lines are fits of the data to Eqs. (2) and (3) with the parameters shown in Fig. 4.

tively. A small initial phase offset, $\phi_{0}$, was observed below $T_{\mathrm{N}}$, larger than could be attributed to errors in determining the time that the muons enter the sample. This could be produced by a small magnetic inequivalency in the position of muons stopped within the sample, consistent with an asymmetric peak seen in Fourier transforms of the data. In the fitting procedure, data were fitted in the time range $0<t<8 \mu s$, where the effect of background counts could be reliably subtracted. Rapid dynamic fluctuations lead to $\lambda_{1}$ $\propto \gamma_{\mu}{ }^{2}(\Delta B)^{2} / \nu$, where $\Delta B$ is the amplitude of the fluctuating local field and $\nu$ is the fluctuation rate. ${ }^{20}$

Spectra measured at four temperatures are shown in Fig. 3. There are three distinct temperature regions apparent from the muon asymmetry spectra. At low temperatures $(T \leqslant 19.5 \mathrm{~K})$ there are clear oscillations in the asymmetry showing that long range magnetic order exists and the observed ratio of $P_{2}: P_{1} \approx 2$ [see Fig. 4(b)] indicates that the sample is magnetic over its entire volume. The values of $\lambda_{2}$ and $\lambda_{\mathrm{s}}$ are much larger than those of $\lambda_{1}$ and $\lambda_{\mathrm{f}}$ [see Figs. 4(c) and $4(\mathrm{~d})$ ], so at short times only the effects of $\lambda_{2}$ and $\lambda_{\mathrm{s}}$ are seen in Fig. 3. An intermediate temperature range $(19.5<T<24 \mathrm{~K})$ gives no oscillations, and the relaxation is modelled with the two exponential components of Eq. (3), with the amplitude of the faster relaxing component decreasing with increasing temperature. Above $24 \mathrm{~K}$ the relaxation is well described by a single exponential, $P_{\mathrm{f}} \exp \left(-\lambda_{\mathrm{f}} t\right)$, consistent with fast fluctuations of paramagnetic moments characterized by a single correlation time in the muon time window.

The temperature dependence of the parameters derived from fitting Eqs. (2) and (3) to muon asymmetry spectra are presented in Fig. 4. The muon precession frequency, $\nu_{\mu}$, in the ordered phase is shown in Fig. 4(a). This is proportional to the sublattice magnetization at the muon site, and was fitted to a function ${ }^{21}$

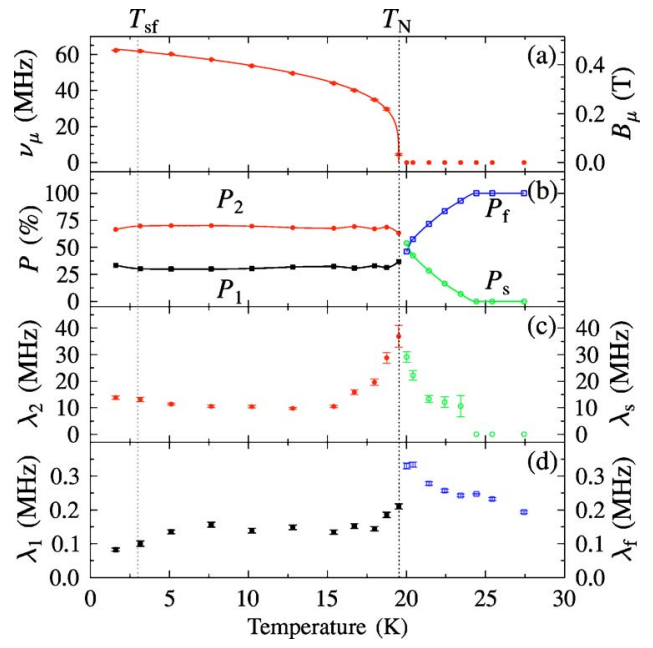

FIG. 4. (Color online) Temperature dependence of the parameters determined from fitting data to Eqs. (2) and (3): (a) the oscillation frequency, $\nu_{\mu}$ and the internal magnetic field, $B_{\mu}$, with a fit to Eq. (4). (b) Amplitudes of the relaxation components $P_{1}$ and $P_{2}$, and $P_{\mathrm{f}}$ and $P_{\mathrm{s}}$. (c) Relaxation rates $\lambda_{2}$ and $\lambda_{\mathrm{s}}$. (d) Relaxation rates $\lambda_{1}$ and $\lambda_{\mathrm{f}}$. The vertical dashed lines indicate temperatures referred to in the text.

$$
\nu_{\mu}(T)=\nu_{\mu}(0)\left[1-\left(T / T_{\mathrm{N}}\right)\right]^{\beta_{\mathrm{m}}} .
$$

The fit gives $\nu_{\mu}(0)=64.2(2) \mathrm{MHz}$ corresponding to a field at the muon site of $\sim 0.5 \mathrm{~T}$. Dipole field calculations show that this field will be experienced by muons near any of the oxygen atoms in the octahedron surrounding a nickel atom, in regions of high electron density, ${ }^{22}$ and show that our results are consistent with the magnetic structure determined by Darie et al. ${ }^{10}$ Our calculations also suggest that the muon precession frequency is insensitive to small deviations from this magnetic structure. Fitting Eq. (4) to the muon precession frequencies gave $T_{\mathrm{N}}=19.51(1) \mathrm{K}$ and $\beta_{\mathrm{m}}=0.24(1)$. This value of $\beta_{\mathrm{m}}$ suggests that the system is behaving as a twodimensional (2D) $X Y$ magnet. ${ }^{23}$

\section{DISCUSSION AND CONCLUSIONS}

From our muon-spin rotation, heat capacity and magnetic susceptibility data we determine $T_{\mathrm{N}}=19.5 \mathrm{~K}$, in agreement with the values determined in Refs. 12-14. We have followed the power law variation of the sublattice magnetization as a function of temperature, which shows strong twodimensionality in the magnetic ordering. The $B-T$ phase diagram presented in Fig. 2(b) shows that the boundary of the AF ordered phase also tends smoothly to $T_{\mathrm{N}}$.

In relation to the peak just above $T_{\text {sf }}$ in the magnetic susceptibility, the formation of a true spin glass is excluded by the observation of muon precession down to $1.6 \mathrm{~K}$. The most plausible interpretation of this feature is a slowing of spin fluctuations around $T_{\mathrm{sf}}$. It is also possible that a small concentration of oxygen vacancies, each with two associated $\mathrm{Ni}^{2+}$ impurity spins for charge balance, exist within the $\mathrm{NiO}_{6}$ layers. These would weakly couple with one another with a separate energy scale to the bulk $\mathrm{Ni}^{3+}$ spins, and given their 
larger spin could account for such an artifact in the magnetic susceptibility. This artifact is unlikely to be related to stacking frustration. We note that extrapolating the Ogielski scaling relation to the muon time window suggests a maximum in the dynamic relaxation rate $\lambda_{1}$ should be observed around $7 \mathrm{~K}$, and a broad maximum of low amplitude is just detectable at this temperature [Fig. 4(d)].

The presence of two exponential relaxation components above $T_{\mathrm{N}}$ [see Eq. (3)] suggests that short-range magnetic order persists over a small temperature range of $\sim 5 \mathrm{~K}$ above $T_{\mathrm{N}}$. The fluctuations in the magnetic field producing the faster relaxing component, $\lambda_{\mathrm{s}}$, are two orders of magnitude slower than those producing the slowly relaxing component, $\lambda_{\mathrm{f}} \cdot P_{\mathrm{s}}$ decreases with increasing temperature up to $24 \mathrm{~K}$, showing that the ratio of slow to fast dynamic relaxation is decreasing. Above this temperature the muon relaxation is that expected for a system in the fast-fluctuation regime. The slow spin relaxations observed in the frequency dependence of the ac susceptibility above $25 \mathrm{~K}$ are not within the muon time window so are not observed. The observation of two components in the muon relaxation below $24 \mathrm{~K}$, together with a changing frequency dependence of the ac susceptibility, suggest a model of coalescing magnetic clusters forming well above $T_{\mathrm{N}}$. On cooling below $\sim 50 \mathrm{~K}$, ferromagnetic clusters form within the $\mathrm{NiO}_{6}$ layers and there will be weak coupling between layers. As the volume fraction of clusters increases with decreasing temperature, the coupling of clusters between layers becomes more significant. This is seen in the increase in the frequency dependence of the ac susceptibility until it reaches a maximum at $\sim 25 \mathrm{~K}$, suggesting that slowly fluctuating short-range order occurs at this temperature. Between $\sim 25 \mathrm{~K}$ and $T_{\mathrm{N}}$ the magnetic clusters coalesce, seen in the decrease of $\chi^{\prime}$ and $\chi^{\prime \prime}$, effectively increasing the registry between planes. The fluctuations in this temperature range are relatively slow, leading to the $P_{\mathrm{s}}$ component of the muon relaxation, and would appear static within the neutron time window. This perhaps explains the $(0,0,1 / 2)$ antiferromagnetic Bragg peaks persisting above $T_{\mathrm{N}}$, as reported in Ref. 11, which would then result from the coalescing clusters of ordered spins.

In conclusion, $\mathrm{NaNiO}_{2}$ shows the onset of long-range magnetic order at $T_{\mathrm{N}}=19.5 \mathrm{~K}$, with the dependence of the sublattice magnetization on temperature appropriate for a $2 \mathrm{D}$ $X Y$ magnet. The slowing of spin fluctuations above $T_{\mathrm{sf}}$ is evident in the ac magnetic susceptibily data. At temperatures just above $T_{\mathrm{N}}$ there is evidence of short-range order and of magnetic clusters persisting within a paramagnetic phase above this temperature.

\section{ACKNOWLEDGMENTS}

Part of this work was performed at the Swiss Muon Source, Paul Scherrer Institute, Villigen, Switzerland. We are grateful to Robert Scheuermann for experimental assistance, and to M. Holzapfel, B. D. Gaulin, and A. Coldea for helpful discussions. T.L. acknowledges support from the European Commission under the 6th Framework Programme through the Key Action: Strengthening the European Research Area, Research Infrastructures. Contract No: RII3-CT2003505925. This work was funded by the EPSRC (UK).
${ }^{1}$ M. F. Collins and O. A. Petrenko, Can. J. Phys. 75, 605 (1997).

${ }^{2}$ R. Coldea, D. A. Tennant, and Z. Tylczynski, Phys. Rev. B 68, 134424 (2003).

${ }^{3}$ B. C. Sales, R. Jin, K. A. Affholter, P. Khalifah, G. M. Veith, and D. Mandrus, Phys. Rev. B 70, 174419 (2004).

${ }^{4}$ S. Kobayashi, S. Mitsuda, and K. Prokes, Phys. Rev. B 63, 024415 (2000).

${ }^{5}$ A.-M. Daré, R. Hayn, and J.-L. Richard, Europhys. Lett. 61, 803 (2003).

${ }^{6}$ F. Vernay, K. Penc, P. Fazekas, and F. Mila, Phys. Rev. B 70, 014428 (2004).

${ }^{7}$ A. J. W. Reitsma, L. F. Feiner, and A. M. Oleś, New J. Phys. 7, 121 (2005).

${ }^{8}$ M. D. Núñez-Regueiro, E. Chappel, G. Chouteau, and C. Delmas, Eur. Phys. J. B 16, 37 (2000).

${ }^{9}$ H. Kikuchi, H. Nagasawa, M. Mekata, Y. Fudamoto, K. M. Kojima, G. M. Luke, Y. J. Uemura, H. Mamiya, and T. Naka, Hyperfine Interact. 120-121, 623 (1999).

${ }^{10}$ C. Darie, P. Bordet, S. de Brion, M. Holzapfel, O. Isnard, A. Lecchi, J. E. Lorenzo, and E. Suard, Eur. Phys. J. B 43, 159 (2005).

${ }^{11}$ M. J. Lewis, B. D. Gaulin, L. Filion, C. Kallin, A. J. Berlinsky, H. A. Dabkowska, Y. Qiu, and J. R. D. Copley, Phys. Rev. B 72, 014408 (2005).
${ }^{12}$ P. F. Bongers and U. Enz, Solid State Commun. 4, 153 (1966).

${ }^{13}$ J. P. Kemp, P. A. Cox, and J. W. Hodby, J. Phys.: Condens. Matter 2, 6699 (1990).

${ }^{14}$ E. Chappel, M. D. Núñez-Regueiro, F. Dupont, G. Chouteau, C. Darie, and A. Sulpice, Eur. Phys. J. B 17, 609 (2000).

${ }^{15}$ E. Chappel, M. D. Núñez-Regueiro, G. Chouteau, O. Isnard, and C. Darie, Eur. Phys. J. B 17, 615 (2000).

${ }^{16}$ M. Holzapfel, S. de Brion, C. Darie, P. Bordet, E. Chappel, G. Chouteau, P. Strobel, A. Sulpice, and M. D. Núñez-Regueiro, Phys. Rev. B 70, 132410 (2004).

${ }^{17} \mathrm{~S}$. Tornow, O. Entin-Wohlman, and A. Aharony, Phys. Rev. B 60, 10206 (1999).

${ }^{18}$ A. T. Ogielski, Phys. Rev. B 32, 7384 (1985).

${ }^{19}$ S. J. Blundell, Contemp. Phys. 40, 175 (1999).

${ }^{20}$ P. Dalmas de Réotier and A. Yaouanc, J. Phys.: Condens. Matter 9, 9113 (1997).

${ }^{21}$ F. Borsa, P. Carreta, J. H. Cho, F. C. Chou, Q. Hu, D. C. Johnston, A. Lascialfari, D. R. Torgeson, R. J. Gooding, N. M. Salem, F. Borsa, P. Carreta, J. H. Cho, F. C. Chou, Q. Hu, D. C. Johnston, A. Lascialfari, D. R. Torgeson, R. J. Gooding, N. M. Salem, K. J. E. Vos, Phys. Rev. B 52, 7334 (1995).

${ }^{22}$ H. Meskine and S. Satpathy, J. Appl. Phys. 97, 10A314 (2005).

${ }^{23}$ S. T. Bramwell, P. C. W. Holdsworth, and M. T. Hutchings, J. Phys. Soc. Jpn. 64, 3066 (1995). 